\title{
Acute and chronic mesenteric ischemia: MDCT findings
}

\author{
Isquemia mesentérica aguda e crônica: achados tomográficos
}

\author{
Bruna Schmitz Serpa, ${ }^{1}$ Adriano Tachibana, ${ }^{2}$ Ronaldo Hueb Baroni, ${ }^{3}$ Ricardo Aun, ${ }^{3}$ Marcelo Buarque Gusmão Funari ${ }^{3}$
}

\begin{abstract}
Mesenteric ischemia is caused by a reduction in mesenteric blood flow. It can be divided into acute and chronic, based upon the rapidity and the degree to which the blood flow is compromised. The authors retrospectively reviewed 22 cases of mesenteric ischemia, diagnosed by multidetector computed tomography (MDCT) in our service, and confirmed by surgery or clinical follow-up. The frequency of the diagnostic findings of chronic and acute mesenteric ischemia was evaluated. The improvement of three-dimensional (3D) MDCT allows accurate assessment of mesenteric vessels. Therefore, it demonstrates changes in ischemic bowel segments helpful in determining the primary cause of the disease, and can identify the complications in patients with acute and chronic mesenteric ischemia.
\end{abstract}

Keywords: Acute mesenteric ischemia, chronic mesenteric ischemia, multi-detector computed tomography.

\section{Resumo}

A isquemia mesentérica é causada pela redução do fluxo sanguíneo mesentérico. Essa patologia pode ser dividida em aguda e crônica, baseada na rapidez e no grau em que o fluxo sanguíneo está sendo comprometido. Os autores retrospectivamente revisaram 22 casos de isquemia mesentérica, diagnosticados por tomografia computadorizada com mutidetectores (TCMD) em nosso serviço, e confirmados por cirurgia ou seguimento clínico. Os achados diagnósticos de isquemia aguda e crônica e sua frequência foram avaliados. Os avanços na TCMD 3D (tridimensional) permitiram o acesso detalhado aos vasos mesentéricos. Além disso, é possível demonstrar alterações nos segmentos intestinais, auxiliando na identificação da causa primária da doença e podendo identificar as complicações associadas a isquemia mesentérica e crônica.

Palavras-chave: Isquemia mesentérica aguda, isquemia mesentérica crônica, tomografia computadorizada com multidetectores.

\section{Introduction}

Mesenteric ischemia is caused by a reduction in blood flow for intestinal circulation of sufficient magnitude to compromise the viability of the affected portion ${ }^{1-5}$.

The disease can be classified into acute and chronic, based upon the rapidity and the degree to which blood flow is compromised and the duration of the symptoms. ${ }^{1-3}$ Historically, the lack of an accurate and readily-available diagnostic imaging tool has played a role in the high morbidity and mortality of this disease $e^{1,6,7}$.

The diagnosis of acute mesenteric ischemia (AMI) is present in nearly $1 \%$ of the acute abdomens, with mortality rates ranging from 59 to $93 \% .{ }^{1,2}$ It can be caused by thromboembolism (in $60-70 \%$ of cases), bowel obstruction, abdominal inflammatory disease and trauma ${ }^{3,4,6}$.
It is important to notice that the computerized tomography (CT) appearance of acute bowel ischemia will depend on its cause, severity, location and extent. The findings can be heterogeneous and nonspecific. The most frequent cause of acute bowel ischemia is thromboembolism, associated with cardiovascular problems. Other acute conditions are aortic and mesenteric dissection, hypercoagulable state, aortic surgery, decreased cardiac output and bowel obstruction ${ }^{1-3,5}$.

There are three stages of acute bowel ischemia. The first stage, which is characterized by mucosal necrosis and mucosal erosion or hemorrhage, is reversible. In stage II, the damage extends to submucosal and muscular layers ${ }^{7}$. Stages I and II are poorly identified on CT. Mucosal enhancement and wall thickening can be detected, but those findings are usually mild and nonspecific ${ }^{7}$. In stage III, which represents transmural bowel wall necrosis, the CT findings are more

MD. Hospital Israelita Albert Einstein (HIAE), São Paulo, Brazil.

${ }^{2}$ MD. HIAE, São Paulo, Brazil. Hospital das Clínicas, Faculdade de Medicina da Universidade de São Paulo (FMUSP), São Paulo, Brazil.

${ }^{3}$ MD. PhD. HIAE, São Paulo, Brazil. Hospital das Clínicas, FMUSP, São Paulo, Brazil.

No conflicts of interest declared concerning the publication of this article.

Manuscript received Apr 20 2009, accepted for publication Nov 262009.

J Vasc Bras. 2010;9(3):156-163. 
reliable and specific, and include abnormal or absent wall enhancement, mesenteric stranding, ascites, pneumatosis and portal venous gas ${ }^{7}$.

Chronic mesenteric ischemia (CMI) affects individuals of advanced age, often in the seventh and eighth decades of life, and with systemic atherosclerosis ${ }^{4,7}$.

The main cause of chronic mesenteric ischemia is atheroma, and the arterial occlusions usually involve proximal segments. Other nonatheromatous conditions include Takayasu arteritis, dysplastic lesions, and thromboangiitis obliterans, among others ${ }^{4,6}$.

The collateral pathways are formed between celiac trunk and superior mesenteric artery through gastroduodenal artery and pancreatic arch, or between superior mesenteric artery and inferior mesenteric artery through marginal artery of the colon. The inferior mesenteric artery anastomoses with the lumbar branches of abdominal aorta and the internal iliac artery. These collateral pathways become extremely prominent and tortuous in chronic mesenteric ischemia ${ }^{4,6,7}$.

The ischemic symptoms depend on the number and site of the lesions, their progression, and the patency of collateral vessels ${ }^{2-6}$.

Angiography is considered the gold-standard for the diagnosis of mesenteric ischemia, but it is an invasive method, can rarely cause morbidity, and does not allow the evaluation of the bowel and other related findings ${ }^{1,3,8}$.

MDCT, especially with newer 16 or more detector rows scanners, has evolved as a self-comprehensive imaging modality of choice for mesenteric ischemia, allowing evaluation of vascular structures, bowel wall and adjacent mesentery, thus determining the primary cause of mesenteric ischemia ${ }^{9-11}$.

\section{Method}

The authors retrospectively reviewed cases of mesenteric ischemia, diagnosed by MDCT in our service, and

Table 1 - CT exam thecnique

\begin{tabular}{ll}
\hline Variables & Used Protocol \\
\hline Oral contrast & $\begin{array}{l}\text { Negative (water) or no oral contrast to allow } \\
\text { visualization of the bowel wall } \\
\text { Intravenous contrast }\end{array}$ \\
& $\begin{array}{l}\text { kg, administered by a power injector at a rate of } \\
3.5-4.0 \mathrm{~mL} / \mathrm{s} .\end{array}$ \\
& $\begin{array}{l}\text { Pre-contrast, arteriographic (timed by automated } \\
\text { Multiphase imaging }\end{array}$ \\
bollimation & $16 \times 2$ mm or $64 \times 0.5$ mm \\
Helical pitch & 53 \\
Gantry rotation time & $0.5 \mathrm{~s}$ \\
kV & 120 \\
mAs & automatic low-dose modulation
\end{tabular}

confirmed by surgery or clinical follow-up during the period of January 2007 until January 2008. The frequency of the diagnostic findings of chronic and acute mesenteric ischemia was observed.

Exams were performed on 16 or 64 detector-rows Aquilion ${ }^{\circledR}$ scanners (Toshiba, Otawara, Japan), using the protocols shown in Table 1.

\section{Results}

\section{Acute mesenteric ischemia}

MDCT findings in acute mesenteric ischemia in our series are summarized in Table 2 and described below:

- Arterial obstruction (thrombus or embolus) can be sometimes detected even in peripheral branches with adequate fast intravenous contrast (Figure 1).

- Arterial dissection can also be identified in the arterial phase and better visualized in some multiplanar reconstructions (Figure 2).

- Bowel wall thickening is the most common finding in acute mesenteric ischemia, but the least specific, and it is due to mural edema and/or hemorrhage. Normal bowel wall thickness range from 3 to $5 \mathrm{~mm}$, but it depends on the degree of distension and can be confused with normal contractions ${ }^{1,3,7}$ (Figure 3 ).

- Bowel distension: Luminal dilatation and/or air-fluid levels are due to interruption of intestinal peristalsis as a response to ischemic injury or irreversible and transmural ischemic damage to the bowel wall, and is usually observed in stage III disease ${ }^{1,3,7}$ (Figure 4).

- Bowel wall attenuation and enhancement: Hypoattenuation of the wall is caused by edema, and can sometimes be seen in mild ischemia. Hypoenhancement is related to severe ischemia or necrosis. Hyperenhancement, on the other hand, is due to hyperemia and hyperperfusion, representing viability of the bowel wall ${ }^{1,3,7}$ (Figure 5).

Table 2 - CT Findings

\begin{tabular}{l|c}
\hline CT findings & AMI - 9 patients (\%) \\
\hline SMA dissection & $3(33.33)$ \\
SMA thrombosis & $1(11.11)$ \\
Bowel wall thickening & $7(77.78)$ \\
Bowel wall enhancement & $4(44.44)$ \\
Bowel distension & $5(55.55)$ \\
Pneumatosis & $3(33.33)$ \\
Pneumoporta & $3(33.33)$ \\
Ischemia of other organ (kidney) & $1(11.11)$ \\
\hline
\end{tabular}

$\mathrm{AMI}=$ acute mesenteric ischemia; $\mathrm{SMA}=$ superior mesenteric artery 

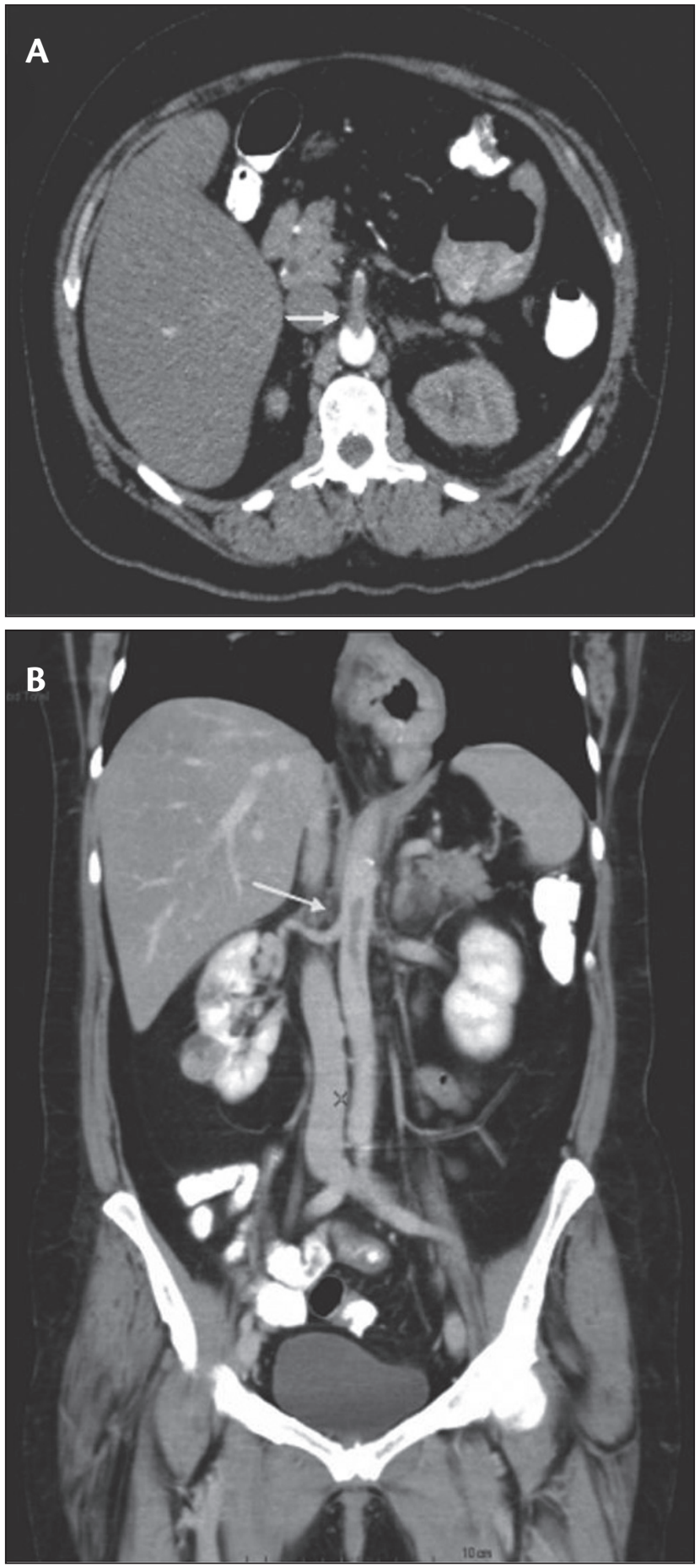

Figure 1 - Occlusive thrombus in superior mesenteric artery. Female, 77 years old. A) axial CT; B) coronal CT

- Pneumatosis: Gas bubbles from the bowel lumen can dissect through the wall. It is a specific but rare finding of acute mesenteric ischemia, and is due to transmural bowel wall necrosis ${ }^{1,3,7}$ (Figure 6).
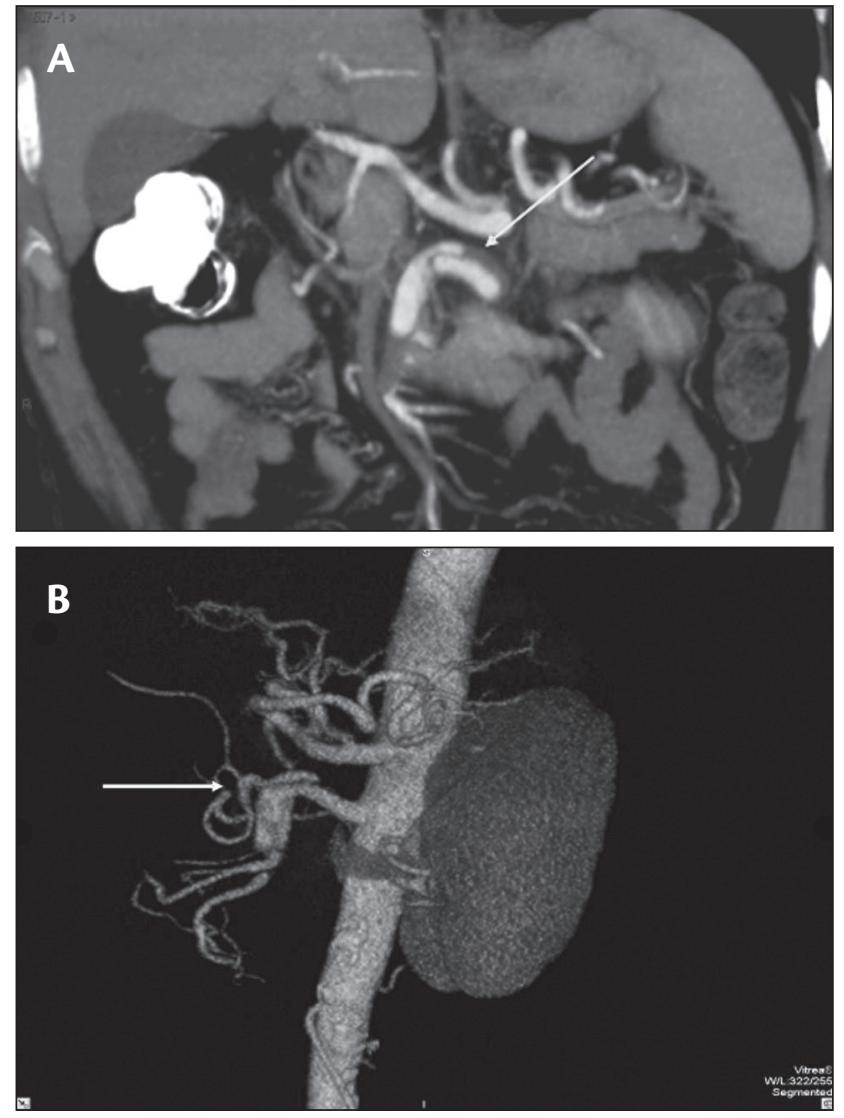

Figure 2 - Superior mesenteric artery dissection. Male, 53 years old: A) coronal CT; B) 3D-MDCT reconstruction

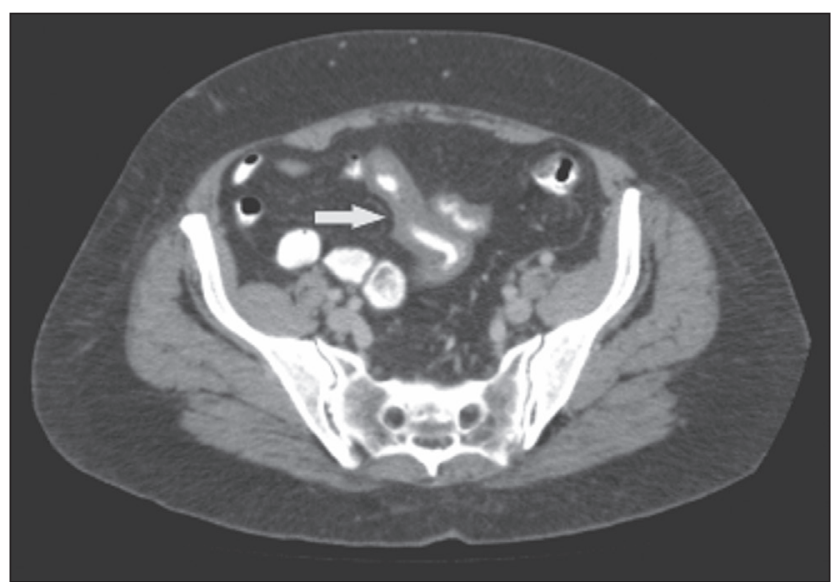

Figure 3 - Axial CT, bowel wall thickening. Female, 77 years old

- Pneumoporta: gas can be restricted to some mesenteric venous branches or may extend to the liver, usually into peripheral portal branches. It is a consequence of pneumatosis $^{1,3,7}$ (Figure 7). 


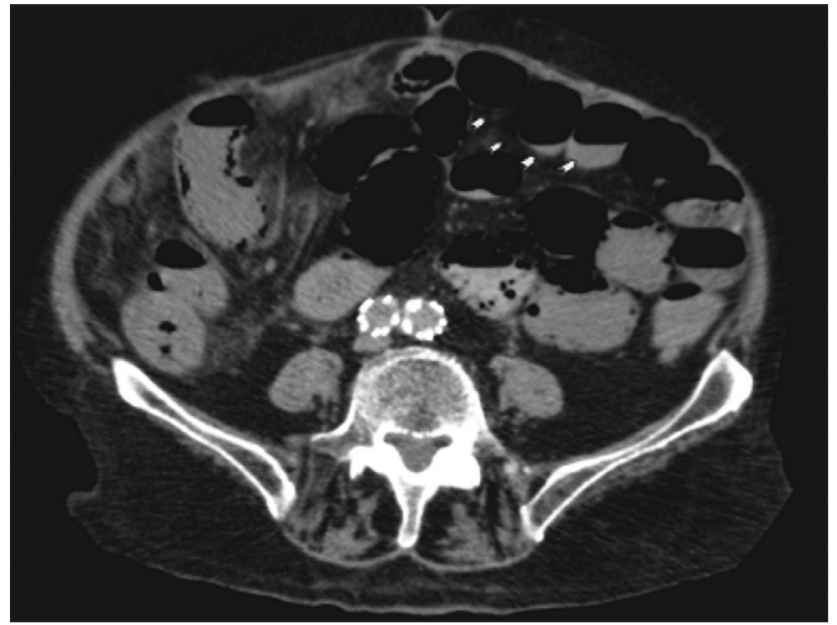

Figure 4 - Axial CT, bowel distension. Male, 66 years old

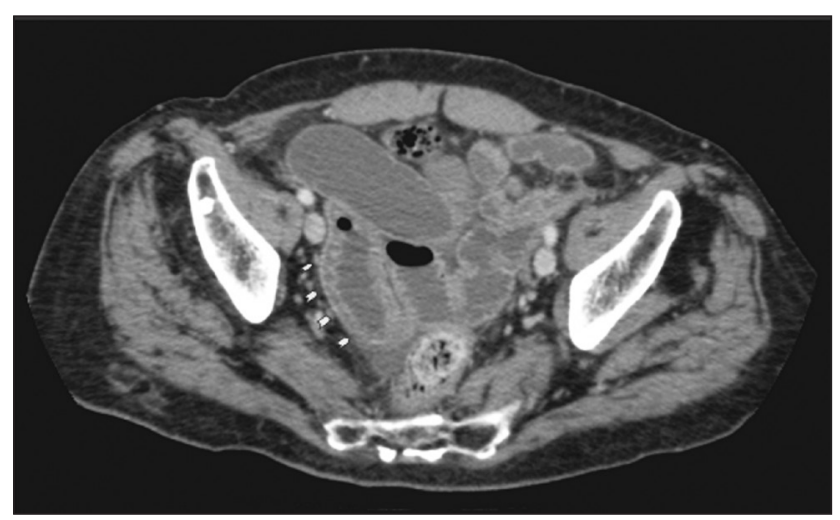

Figure 5 - Axial CT, bowel wall hyperenhancement. Male, 77 years old

\section{Chronic mesenteric ischemia}

MDCT findings in chronic mesenteric ischemia in our series are summarized in Table 3 and further described.

The most common findings of chronic mesenteric ischemia are celiac trunk stenosis (Figure 8), superior mesenteric artery stenosis (Figure 9), inferior mesenteric artery stenosis and collateral pathways: pancreatic arch (Figure 10) and marginal artery of the colon (Figure 11).

Chronic mesenteric ischemia is in the majority of cases associated with superior mesenteric artery stenosis ( $76 \%$ of our cases). It is also commonly combined with stenosis of the celiac trunk and/or the inferior mesenteric artery.

The collateral pathways formed between celiac trunk and superior mesenteric artery through gastroduodenal artery and pancreatic arch, or between superior mesenteric artery and inferior mesenteric artery through marginal artery of the colon, are extremely prominent and tortuous.
Table 3 - CT findings

\begin{tabular}{lc}
\hline CT findings & CMI - 13 patients (\%) \\
\hline Stenosis of celiac trunk & $4(30.76)$ \\
Stenosis of superior mesenteric artery & $10(76.92)$ \\
Stenosis of inferior mesenteric artery & $7(53.84)$ \\
Collateral pathway: pancreatic arch & $13(100)$ \\
Collateral pathway: marginal artery of the colon & $13(100)$ \\
\hline
\end{tabular}

\section{Discussion}

The diagnosis of AMI remains a clinical challenge. The high morbidity and mortality of the disease requires a safe, fast and effective method for the assessment and diagnosis of this entity. Contrast-enhanced CT is an important diagnostic tool, including 3D and multiplanar reconstruction $\mathrm{s}^{1-5}$.

The most prevalent CT findings were related to bowel wall, including thickening, distension and hyperenhancement. Thickening of the bowel wall is the most frequent CT finding pertaining to $\mathrm{AMI}^{1,3}$. This finding was observed in 7 of 9 patients diagnosed with acute mesenteric ischemia (77\% of prevalence). Distension was found in $55 \%$ of the cases. The bowel wall can appear with low attenuation due to swelling and inflammation or with high attenuation due to bleeding in the submucosa. After intravenous injection of contrast, the wall of the loops may appear with low attenuation affected by the compromising of blood flow and with high attenuation due to hyperemia ${ }^{1}$. In our series, $4(44 \%)$ patients had a high attenuation of the bowel wall after the infusion of the contrast, which should correspond to hyperemia, featuring an early stage of injury.

However, those findings are nonspecific responses of the bowel to a wide variety of pathologies, so they lack specificity.

Other findings, directly related to the decrease in blood flow or its late consequences, can also be observed. This will add specificity to the acute mesenteric ischemia diagnosis. These findings are related to occlusion of the splanchnic arteries (celiac trunk, superior mesenteric artery and inferior mesenteric artery) due to thrombus or dissection ${ }^{2,3,7}$. The findings are easily identified during arterial phase of intravenous contrast ${ }^{5}$.

In the AMI group of our study, superior mesenteric dissection was observed in $33 \%$ of the cases, which was a rarely seen event in the literature review. Other specific findings are superior mesenteric artery thrombosis and thromboembolism, which were much more frequent in the literature than in our series. These contradictory numbers 

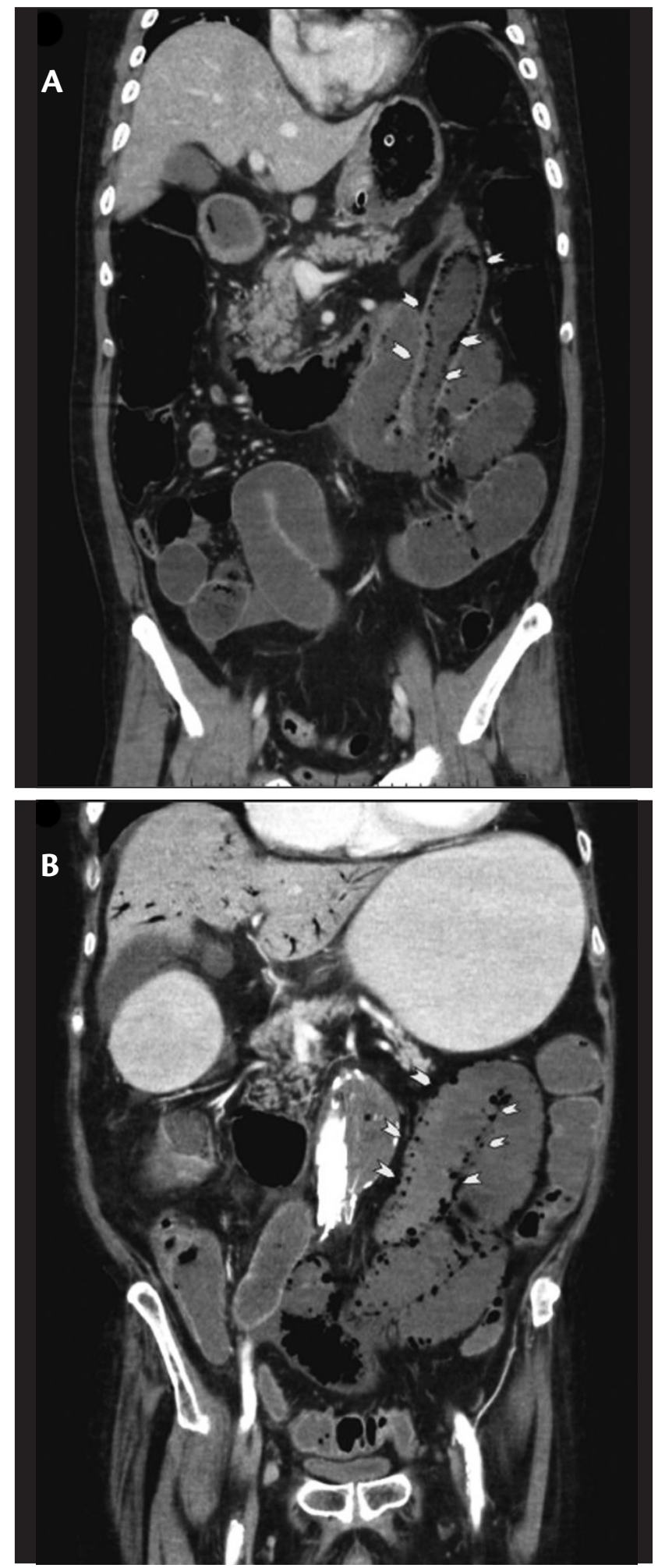

Figure 6 - Coronal CT, pneumatosis: A) male, 65 years old; B) male, 85 years old
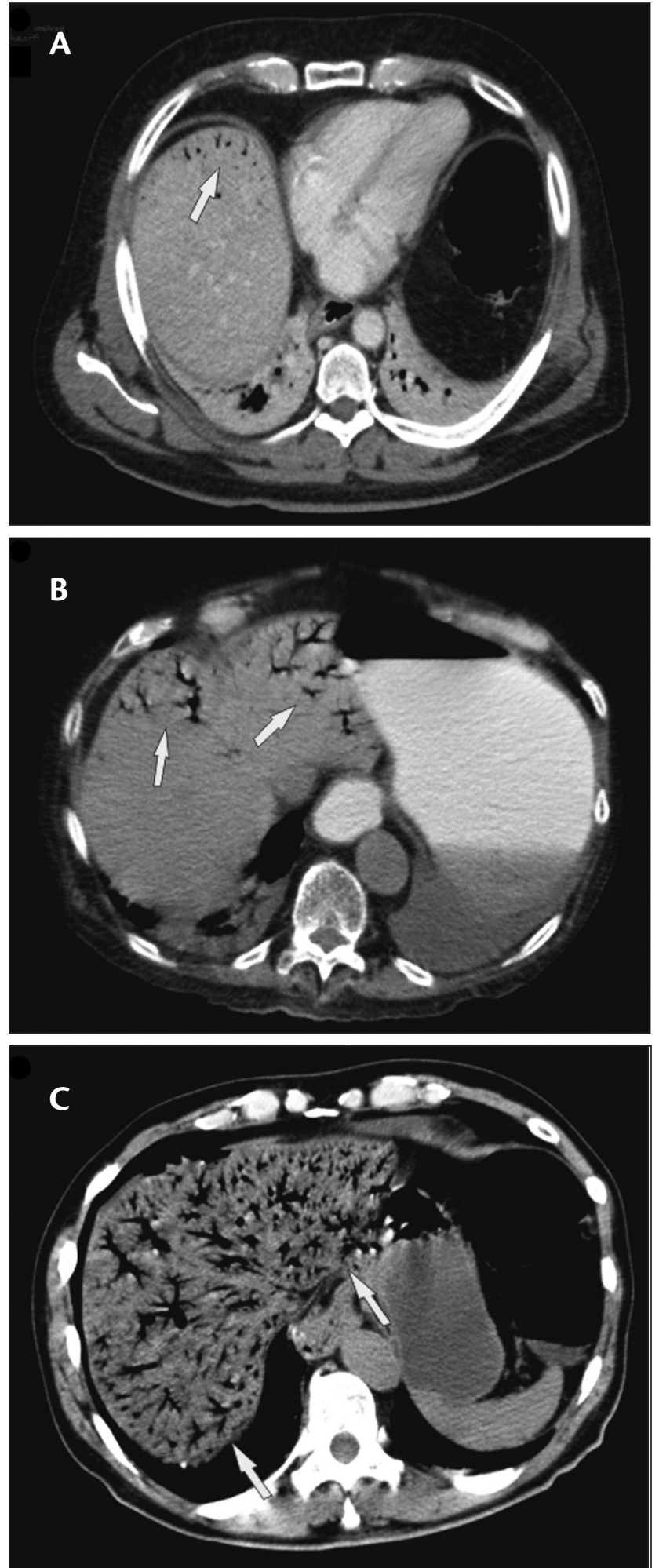

Figure 7 - Axial CT, pneumoporta: A) male, 65 years old; B) male, 85 years old; C) male, 66 years old 


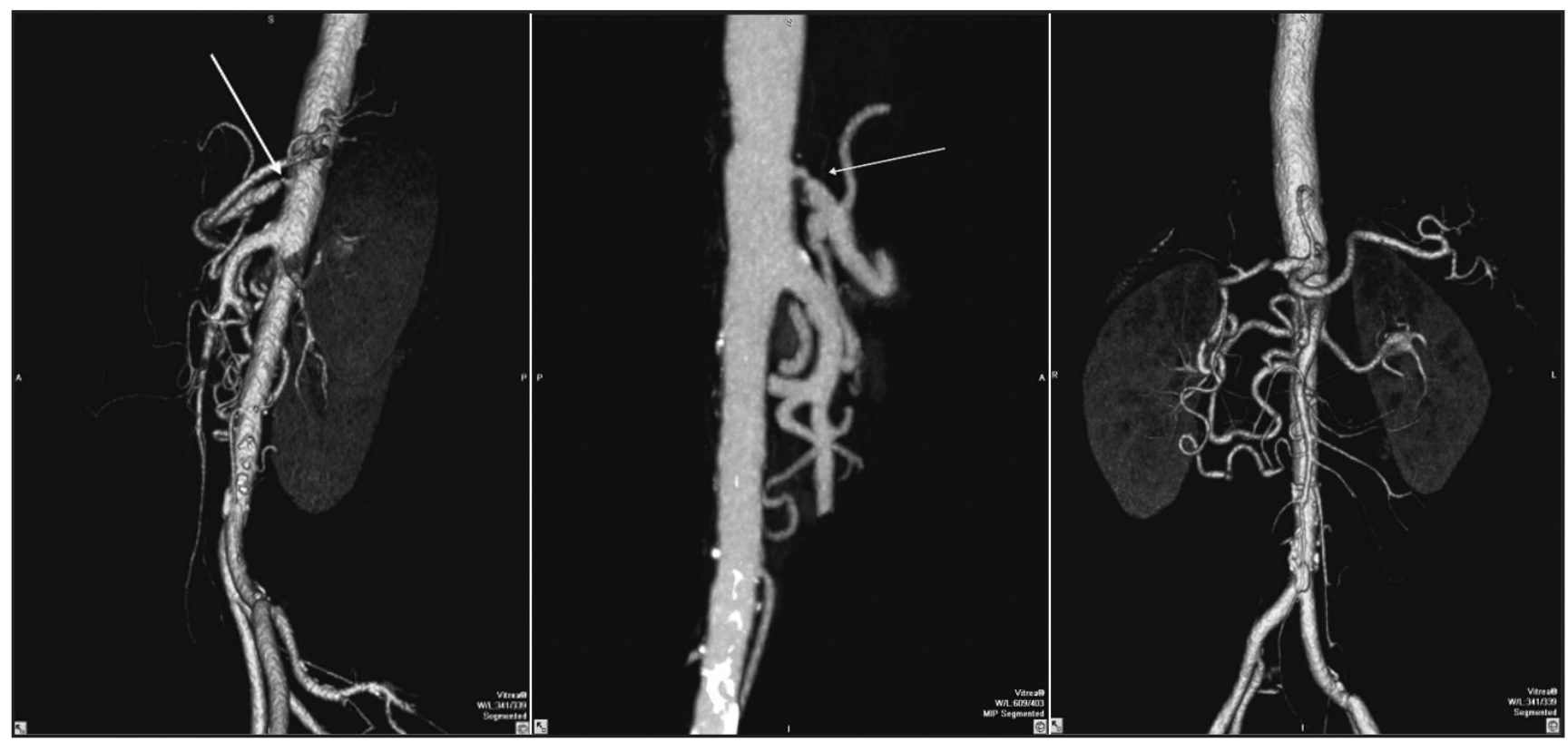

Figure 8 - 3D-MDCT reconstruction. Female, 65 years old, stenosis of celiac trunk

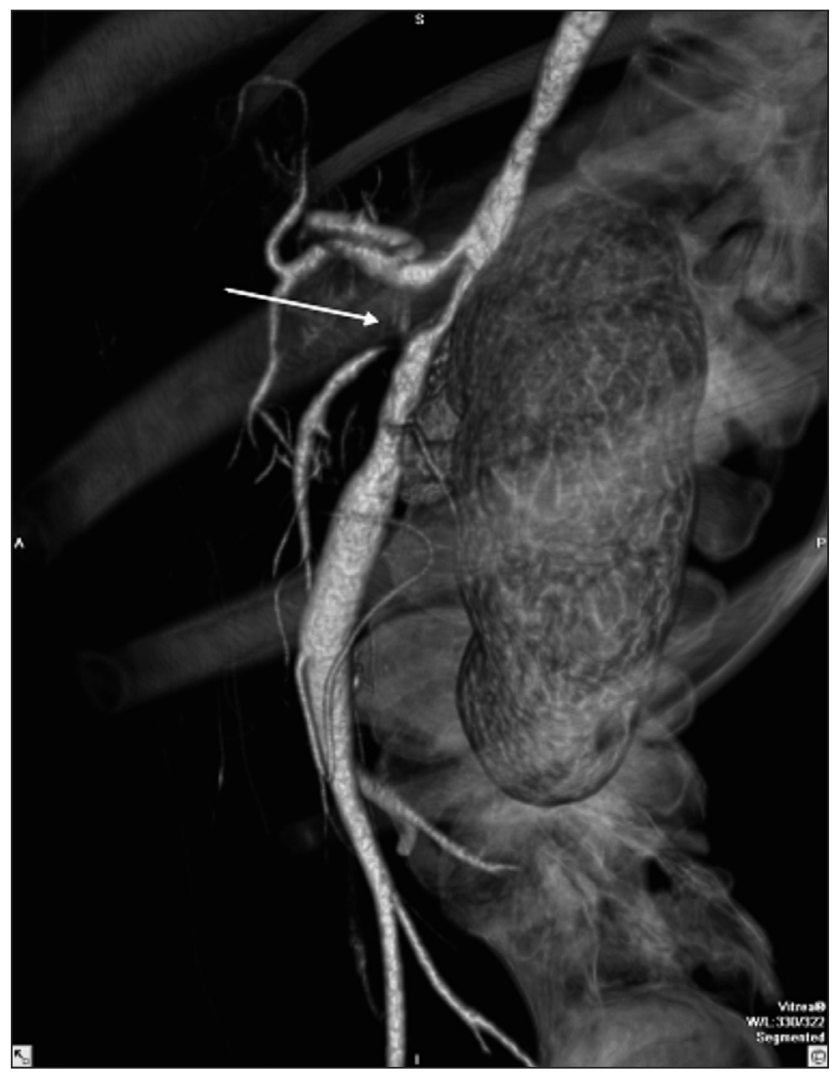

Figure 9 - 3D-MDCT reconstruction. Female, 31 years old, Takayasu arteritis, stenosis of superior mesenteric artery

could be explained by the small group of patients included in our study. The only case of superior mesenteric artery thrombosis was associated with the injury of another target organ (segmental ischemia of the left kidney), suggesting an embolic disease as etiology of the AMI.

The set of findings about AMI in MDCT reformatted images aided by the 3D method provides a sensitivity of 96 and specificity of $94 \%$, according to Kirkpatrick et al $^{1}$.

Bowel wall necrosis with pneumatosis and pneumoporta are extremely specific late onset findings, but only observed in severe cases or in delayed diagnosis, rendering the framework irreversible ${ }^{1,2,7}$. In our study only 3 of 9 patients had pneumatosis.

Chronic mesenteric ischemia presents itself with a more indolent course. The typical form of presentation is recurrent abdominal postprandial pain. The main cause of that entity is the atherosclerotic plaque, which is slowly formed. Since it is a slow process, the affected patients develop collateral vessels in an attempt to maintain an appropriate intestinal perfusion. The symptoms appear when there are no sufficient collateral vessels ${ }^{2,4}$.

Chronic mesenteric ischemia is, in the majority of cases, associated with superior mesenteric artery stenosis ( $76 \%$ of our cases). It is also commonly combined with stenosis of the celiac trunk and/or the inferior mesenteric artery. Cognet et al. ${ }^{4}$ stated that at least two of these three arteries should be stenotic to be the cause of the symptomatology. In our series, only two symptomatic patients had only one artery stenosis.

Hence, the diagnosis of CMI is based on the finding of the stenotic artery, usually accompanied by collaterals, in a compatible clinical context, and when the AMI findings were excluded. 

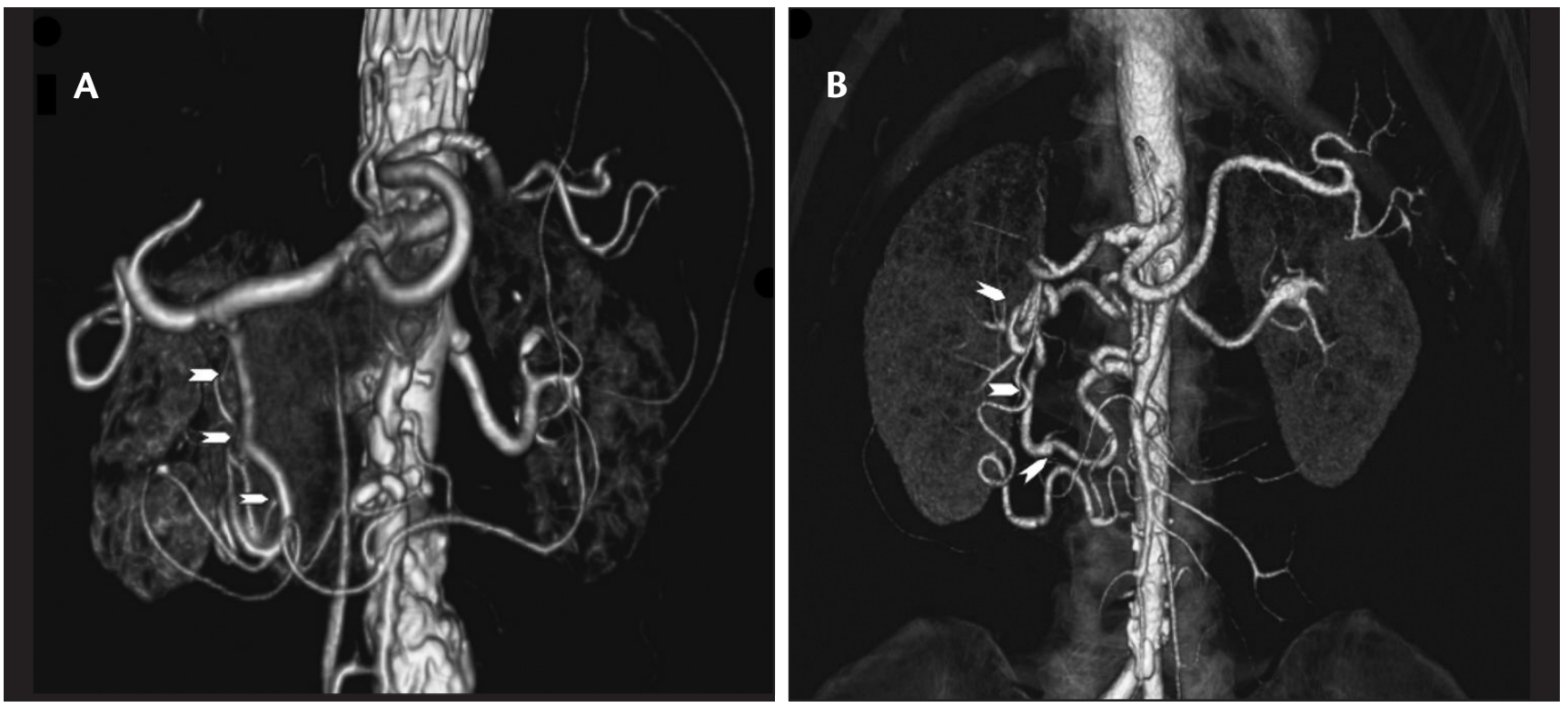

Figure 10 - 3D-MDCT reconstruction, pancreatic arch: A) male, 59 years old; B) female, 65 years old
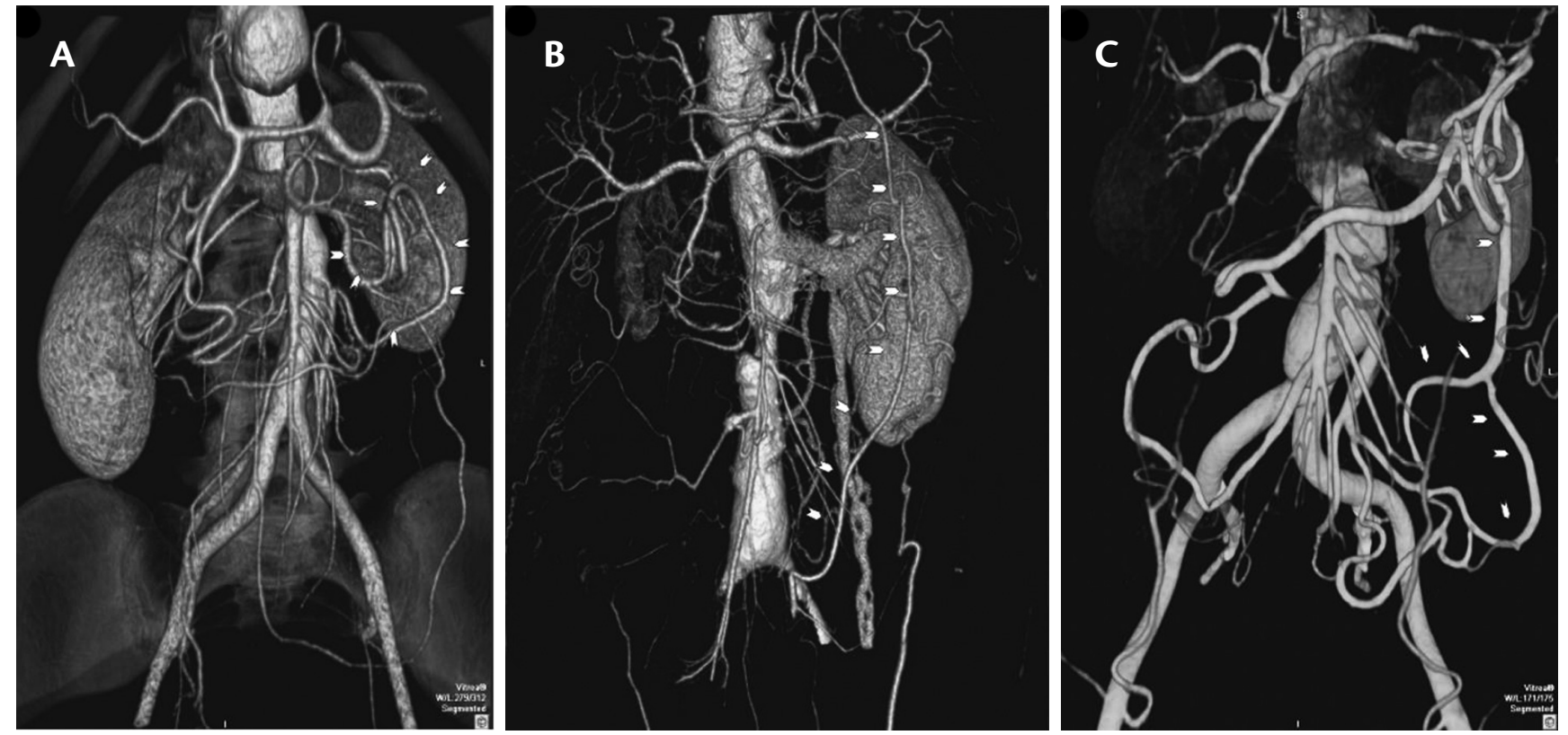

Figure 11 - 3D-MDCT reconstruction, marginal artery of the colon: A) female, 55 years old; B) male, 42 years old with Takayasu arteritis; C) female, 70 years old

CT detects the artery calcification in patients with chronic mesenteric ischemia, but is not a specific finding, since it is extremely common in elderly patients. Significant stenosis of the main vessels (celiac trunk, superior mesenteric artery and inferior mesenteric artery) can be identified ${ }^{2,4-6}$.

In this study the strictures of celiac trunk were identified in 4 patients, the superior mesenteric artery in 10 patients and inferior mesenteric artery in 7 of 13 patients evaluated.
In CMI, usually there are no morphologic changes in the intestinal loops. The most common finding on CT is the prominent collateral vessels, which are, in the majority of cases, tortuous ${ }^{2,4,5}$. In our study, all patients diagnosed with CMI (13) had prominent pancreatic arcade and marginal artery of the colon. These findings were better evaluated at arterial phase of CT through 3D reconstructions.

There are some limitations in our study; among which the most important was the small number of cases, since 
mesenteric ischemia is not a common entity. Other important point was that not all cases were surgically confirmed, although clinical findings were considered sufficient for the diagnosis.

AMI and CMI are diseases with high morbidity and mortality rates, and a readily-available and accurate diagnostic imaging tool is essential for a fast diagnosis and treatment. MDCT has evolved as a self-comprehensive imaging modality of choice for mesenteric ischemia, allowing the evaluation of vascular structures, bowel wall and adjacent mesentery, thus determining the primary cause of mesenteric ischemia.

\section{References}

1. Kirkpatrick IDC, Kroeker MA, Greenberg HM. Biphasic CT with mesenteric CT angiography in the evaluation of acute mesenteric ischemia: initial experience. Radiology. 2003;229:91-8.

2. Horton KM, Fishman EK. Multi-detector row CT of mesenteric ischemia: can it be done? RadioGraphics. 2001;21:1463.

3. Taourel PG, Deneuville M, Pradel JA, Regent D, Bruel JM. Acute mesenteric ischemia: diagnosis with contrast-enhanced CT. Radiology. 1996;199:632-6.

4. Cognet F, Ben Salem D, Dranssart M, et al. Chronic mesenteric ischemia: imaging and percutaneous treatment. RadioGraphics. 2002;22:863-79.

5. Horton KM, Fishman EK. Volume-rendered 3D CT of the mesenteric vasculature: normal anatomy, anatomic variants, and pathologic conditions. RadioGraphics. 2002;22:161.

6. Rha SE, Ha HK, Lee SH, et al. CT and MR imaging findings of bowel ischemia from various primary causes. RadioGraphics. 2000;20:29.
7. Wiesner W, Khurana B, Ji H, Ros PR. CT of acute bowel ischemia. Radiology. 2003;226:635-50.

8. Shih MCP, Hagspiel KD. CTA and MRA in mesenteric ischemia: part 1, role in diagnosis and differential diagnosis. Am J Roentgenol. 2007;188:452-61.

9. Shih MCP, Angle JF, DA Leung, et al. CTA and MRA in mesenteric ischemia: part 2, normal findings and complications after surgical and endovascular treatment. Am J Roentgenol. 2007;188:462-71.

10. Zalcman M, Sy M, Donckier V, Closset J, Van Gansbeke D. Helical CT signs in the diagnosis of intestinal ischemia in small-bowel obstruction. Am J Roentgenol. 2000;175:1601-7.

11. Chen JK, Johnson PT, Horton KM, Fishman EK. Unsuspected mesenteric arterial abnormality: comparison of MDCT axial sections to interactive 3D rendering. Am J Roentgenol. 2007;189: 807-13.

Correspondence: Bruna Schmitz Serpa Av. Rouxinol 77/93 CEP 04516-000 - São Paulo, SP, Brazil Tel.: +55 (11) 86353793, +55 (11) 35820432 E-mail: bruna.serpa@gmail.com

Author contributions Conception and design: AT, RHB, BSS Analysis and interpretation: BSS Data collection: BSS Writing the article: BSS, AT Critical revision of the article: $A T$, RHB, RA Final approval of the article*: RA, AT, RA Statistical analysis: BSS, RHB Overall responsibility: BSS Obtained funding: BSS * All authors have read and approved of the final version of the article submitted to I Vasc Bras. 